\title{
Richtigstellung: Saphenofemorale Rezidive nach endovenöser thermischer Therapie und nach chirurgischer Therapie
}

\section{Thermische Ablation kosten- günstiger als chirurgische Operation!}

Die Arbeitsgruppe Mumme, Mühlberger, Sidhwa und Hummel haben mit ihrer Arbeit „Alarmierend hohe Rate saphenofemoraler Rezidive nach endovenöser Lasertherapie“ (Phlebologie 2019;48:18-22) mehrere Behauptungen aufgestellt, die nochmals genauer beleuchtet werden müssen.

\section{Saphenofemorales Rezidiv}

Schauen wir uns beispielsweise die SameSite-Rezidive an, die mittels Duplexsonographie nachgewiesen werden können, so sind die von der Arbeitsgruppe Mumme veröffentlichten Vergleichsdaten ( $\triangleright$ Tab. 1) auch so zu lesen, dass es eine unglaubliche Varianz im Auftreten von saphenofemoralen Rezidiven nach Crossektomie gibt (von 5\% Lawaetz, et al. bis 23 \% Gauw et al.). Angesprochen auf diese Diskrepanz, wird von chirurgischer Seite immer angeführt: „Ja, die haben eigentlich gar keine korrekte Crossektomie durchgeführt, sondern schlecht operiert“. Fischer et al. haben z. B. $60 \%$ saphenofemorale Rezidive 34 Jahre nach Crossektomie beschrieben (Fischer et al. 2001)!

Nun ist es so, dass auch die thermische Ablation unterschiedlich genau durchgeführt werden kann. Auch sind die von der Arbeitsgruppe Mumme vorgelegten Stu-

- Tab. 1 Duplexsonographisch detektierbare Crossenrezidive in prospektiven Studien mit Randomisierung und 5-Jahres-Follow-Up. Endovenöse Laserablation (EVLA) im Vergleich mit Crossektomie und Stripping $(\mathrm{C}+\mathrm{S})$.

\begin{tabular}{|l|l|l|l|}
\hline & n & EVLA & C+S \\
\hline Rasmussen 2013 & $58 / 60$ & $8 \%$ & $4 \%$ \\
\hline Van der Velden 2015 & $78 / 69$ & $22 \%$ & $13 \%$ \\
\hline Rass 2015 & $185 / 161$ & $28 \%$ & $5 \%$ \\
\hline Flessenkämper 2016 & $142 / 159$ & $40 \%$ & $10 \%$ \\
\hline Gauw 2016 & $61 / 60$ & $49 \%$ & $23 \%$ \\
\hline Lawaetz 2017 & $144 / 142$ & $5 \%$ & $5 \%$ \\
\hline
\end{tabular}

dien alle mit den alten Lasersystemen mit Bar-Fiber und $980 \mathrm{~nm}$ durchgeführt worden. Mit den neueren Lasersystemen und vor allem der Radialfaser, die einen Abstrahlwinkel von $\sim 180^{\circ}$ aufweist, kann wesentlich akkurater behandelt und die Länge des „Crossenstumpfes“ auf unter $10 \mathrm{~mm}$ beschränkt werden. Wie alle chirurgisch tätigen Kollegen wissen, mündet im saphenofemoralen Bereich von $1 \mathrm{~cm}$ in $99 \%$ der Fälle nur die V. epigastrica und ggf. die V. s. acc. anterior (VSAA) ein. Oder die V.s. magna (VSM) hat einen Verlauf über die VSAA und im Mündungsbereich findet man eine dünne VSM, oder auch V.s. acc. post (VSAP) genannt (obwohl diese eigentlich erst weiter distal in die VSM einmündet) mit medio-posteriorem Verlauf. Es mehren sich Hinweise, dass die Hauptursache eines saphenofemoralen Rezidivs eine belassene VSAA darstellt (Proebstle 2015, Schuler 2018). Der ver- sierte endovenöse Operateur kann problemlos die VSAA und ggf. auch die VSAP in gleicher Sitzung, in der die VSM thermisch verschlossen wird, mitbehandeln, was dann als „endovenöse Crossektomie“ bezeichnet wird (Hartmann et al. 2017). Der Hinweis von Mumme et al., dass die Hitzeablation im proximalen $1-2 \mathrm{~cm}$ Segment der Stammvene verboten ist, ist in manchen (und nicht allen!) Anwendungshinweisen nur deshalb erwähnt, damit unerfahrene Anwender nicht die thermische Sonde in tiefe Venen vorschieben und diese behandeln. Die Energieabgabe der neueren endovenösen thermischen Verfahren beträgt nur noch $60-140^{\circ} \mathrm{C}$. Die Elektrokaustik, welche bei der Operation benützt wird, um die Neoangiogenese zu verhindern und Blutungen zu stoppen, wird auch im 1-2 cm Bereich der saphenofemoralen Junktion verwendet und ist über $1000^{\circ} \mathrm{C}$ heiß! 
Außerdem gibt es die Neoangiogenese, welche nach Stripping-Operationen auftreten kann, nach endovenöser Therapie nicht, da kein freies Endothel zurückbelassen wird. Duplexsonographisch detektierte Crossenrefluxe, die nach endovenöser thermischer Crossektomie mit der Radialfaser (!) auftreten sind entweder bedingt durch eine belassene und jetzt refluxive VSAA, ein (extrem seltenes) Rezidiv aus einer V. pudenda externa profunda (welche direkt in die V. femoralis im Crossenbereich einmündet) oder eine primäre avalvuläre variköse Anomalie (VASA) (Whiteley 2018). Die in - Tab. 1 der Arbeitsgruppe von Mumme aufgeführten Rezidivraten nach endovenöser Lasertherapie sind entweder durch Rekanalisationen bei zu geringer Energie pro Zentimeter Vene verursacht, durch das Verwenden alter Lasersysteme mit Bar Fiber oder durch zu lang belassene Crossenstümpfe über $2 \mathrm{~cm}$ Länge, wie dies im angloamerikanischen Raum die Regel ist.

Nun wird in der Arbeit von Mumme et al. immer die Leistenrezidivstudie erwähnt, welche 2009 veröffentlicht wurde (Mumme et al. 2009). Demnach begünstigt der Verzicht auf eine Crossektomie die Ausbildung von Crossenrezidiven. Ferner schreiben die Autoren, dass eine schlechte Voroperation mit zu lang belassenem Crossenstumpf ein Rezidiv begünstigt und diese „schlechten Voroperationen “ gar nicht so selten in Deutschland vorkommen würden (und übrigens auch weltweit, denn womit wäre sonst erklärbar, dass weltweit die endovenösen thermischen Therapien als 1. Wahl gelten und nicht die Stripping-Operation? [siehe auch die Nice Guidelines]) Eine chirurgische Rezidiv-Operation ist aber im allgemeinen kostspieliger (da vorwiegend stationär durchgeführt), belastender für den Patienten und mit mehr Nebenwirkungen behaftet als ein endovenöses Rezidiv, welches meistens erneut endovenös angegangen werden kann, vor allem ambulant durchgeführt wird und eine geringe Nebenwirkungsrate aufweist.

\section{Kosten}

Auch bezüglich der Kosten liegen die Autoren um Mumme et al. falsch. Eine endovenöse thermische Operation erfolgt fast immer ambulant, die hohen Kosten eines stationären Eingriffs können eingespart werden. Immer noch gibt es in Deutschland viele Venenkliniken, die stationär Stripping-Operationen durchführen und dadurch hohe Kosten für das Gesundheitssystem generieren. Als Beispiel kann unser Venenzentrum in Freiburg dienen: bis vor 5 Jahren hatten wir noch 4 stationäre OPTage mit mit bis zu 40 stationären Stripping-Operationen pro Woche. Jetzt mit den endovenösen Verfahren sind es nur noch bis zu 6 stationäre Stripping-Operationen pro Woche. Auch die von Mumme et al. gern erwähnten teuren Rezidiv-Operationen können heutzutage mittels modernster endovenöser Lasertechnik ambulant und kostengünstig operiert werden. Nehmen wird nun das Kostenbeispiel von Mumme et al. von 64 Mio $€$ Kosten für Rezidivoperationen im Jahr 2000 und einer Kostensteigerung mit dem Faktor 4 bzw. 5,6. Es wundert den Leser, dass Mumme et al. in ihrer Arbeit nur die beiden Studien als Grundlage mit der größten Differenz in den Ergebnissen zur Berechnung des Faktors genommen haben (Rass und Flessenkämper). Wird der Mittelwert aus den 6 Studien genommen, ergibt sich ein Steigerungsfaktor von 2,5. Auch wenn nicht mit einer Steigerung der Rezidivrate bei Durchführung einer endovenösen Crossektomie zu rechnen ist, führen wir das Rechenbeispiel fort und multiplizieren die Kosten einer Rezidivoperation im Jahr 2000 mit 2,5 und erhalten hochgerechnet 160 Mio $€$ Kosten für Rezidivoperationen heutzutage. Eine Rezidivoperation stationär kostet ca. $2000 €$, eine endovenöse Rezidivoperation ambulant kostet ca. $1500 €$ also $1 / 4$ weniger. Somit ergeben sich Gesamtkosten von 40 Mio $€$ heutzutage für Rezidivoperationen, eine Reduktion von $37,5 \%$ ! Letztendlich darf auch nicht der sozioökonomische Aspekt vergessen werden: Nach einer Stripping-Operation sind die meisten Patienten mindestens 10 Tage krankgeschrieben. Nach endovenöser thermischer Operation ist eine Arbeitsunfähigkeit von 1-3 Tagen die Regel und der Patient kann schneller wieder seinen alltäglichen Belangen nachgehen und zum Bruttoinlandsprodukt beitragen.

Demnach sind die endovenös thermischen Operationen und Rezidivoperationen kostengünstiger als der chirurgische Gegenpart!
Aus Sicht der Patienten kann auf die aktuelle Studienlage nur eingegangen werden, wenn die unterschiedlichen Vorgehensweisen bei der Stripping-Operation und bei den endovenösen thermischen Verfahren international und national eingehend erläutert werden, was in einem normalen ArztPatienten-Gespräch zu weit führen würde.

Daher muss dem Patienten gesagt werden, dass wenn ein Crossenstumpf unter $1 \mathrm{~cm}$ bei der endovenösen thermischen Crossektomie zurückbleibt und das Ergebnis hinsichtlich der Rezidivfreiheit mit der korrekt (!) durchgeführten chirurgischen Crossektomie verglichen wird, vermutlich ähnliche Rezidivraten resultieren, es fehlen aber noch vergleichende Studien. Da beide Verfahren (chirurgisch und endovenös thermisch) nun gleich gut bezüglich des Outcomes sind, sollte der Patient nicht zu einer Selbstzahlerleistung der endovenösen Verfahren gedrängt werden. Allerdings muss der Patienten darauf hingewiesen werden, dass er mit der endovenösen thermischen Therapie am nächsten Tag seinen normalen körperlichen Aktivitäten nachgehen und auch arbeiten kann, er keinen Leistenschnitt (mit Fadenzug in 10 Tagen) hat und er mit weniger postoperativen Nebenwirkungen zu rechnen hat.

Literatur

[1] Fischer R, Linde N, Duff C et al. Late recurrent saphenofemoral junction reflux after ligation and stripping of the greater saphenous vein. J Vasc Surg 2001; 34(2): 236-240

[2] Hartmann K, Stenger D, Hartmann M et al. Endovenous ablation versus open surgery for varicose veins: An attempt at an evaluation. Hautarzt 2017; 68 (8): 603-613

[3] Schuler L, Weingard I, Kiderlen $\mathrm{M}$ et al. Endoluminal Thermal Ablation of Varicose Great Saphenous Vein - A Randomized Single Center Application Comparison of Laser Ablation (EVLA 1470nmTM), Radio Frequency Ablation (RFITT and ClosureFastTM) and Superheated Steam with Average Post-Operative Follow-up of 3.8 Years. Annals of vascular medicine and research 2018; 5 (2): 1091

[4] Mumme A, Mühlberger D, Sidhwa Y et al. Alarmierend hohe Rate saphenofemoraler Rezidive nach endovenöser Lasertherapie. Phlebologie 2019; 48: 18-22

[5] Mumme A, Hummel T, Burger $P$ et al. Die Krossektomie ist erforderlich! - Ergeb- 
nisse der Deutschen Leistenrezidivstudie. Phlebologie 2009; 38: 99-102

[6] Proebstle TM, Möhler T. A longitudinal single-center cohort study on the prevalence and risk of accessory saphenous vein reflux after radiofrequency segmental thermal ablation of great saphenous veins. In: Journal of vascular surgery. Venous and lymphatic disorders 2015; 3 (3): 265-269

[7] Whiteley AM, Holdstock JM, Whiteley MS. Symptomatic recurrent varicose veins due to primary avalvular varicose anomalies (PAVA): A previously unreported cause of recurrence. SAGE Open Med Case Rep 2018; 6: doi: 10.1177/2050313X18777166
Sponsoringeinnahmen von Firmen endovenöser Verfahren und von Firmen für

OP-Materialien zur Durchführung des Berliner/Freiburger Venenworkshops.

Autorinnen/Autoren

Dr. med. K. Hartmann

Venenzentrum Freiburg

www.venenzentrum-freiburg.de

Schatzmeister der Deutschen Gesellschaft

für Phlebologie (DGP)

und des Berufsverbandes der

Phlebologen (BVP)

E-Mail: kahartmann@web.de

\section{Publikationshinweis}

Leserbriefe stellen nicht unbedingt die Meinung von Herausgebern oder Verlag dar. Herausgeber und Verlag behalten sich vor, Leserbriefe nicht, gekürzt oder in Auszügen zu veröffentlichen.

\section{Bibliografie}

DOI https://doi.org/10.1055/a-0890-8931 Phlebologie 2019; 48: 193-195

(c) Georg Thieme Verlag KG Stuttgart · New York ISSN 0939-978X 